CRfTICA, Revista Hispanoamericana de Filosofta

Vol. XXII, No. 65 (agosto 1990): 57-72

\title{
LA JUSTIFICACIÓN PRÁCTICA DEL PRINCIPIO DE NO CONTRADICCIÓN EN ARISTÓTELES
}

ALEJANDRO CASSINI

Universidad de Buenos Aires

En Metafisica IV, 4, 1008 b 12-31 Aristóteles apela a un argumento de carácter práctico con el fin de mostrar que nadie niega de hecho el principio de no contradicción (en adelante abreviado como PNC). Emplea para ello consideraciones acerca de la acción humana. Según su punto de vista, la práctica permite comprobar que las acciones de los hombres nunca son ejecutadas con base en creencias contradictorias. En la acción se muestra, entonces, que nadie cree efectivamente que dos enunciados de la forma $P a$ y $-P a$ sean simultáneamente verdaderos. Aunque alguien diga que tal contradicción es verdadera, no actúa como si creyera que lo es, es decir, sus acciones no se ajustan a esta creencia contradictoria. La interpretación de los hechos de la práctica es capaz de exhibir la adecuación que existe entre la acción humana y la consistencia de las creencias. Así, de acuerdo con Aristóteles, a partir del análisis de lo observado en las acciones humanas es posible encontrar una nueva razón que apoye la admisibilidad del PNC.

Los aspectos fundamentales del argumento aristotélico se concentran en el siguiente pasaje: “¿Por qué (alguien) camina hacia Megara en vez de quedarse quieto, cuando considera que debe caminar? ¿Por qué una mañana no se dirige pronto hacia un pozo o hacia un precipicio, si por casualidad (halla uno), en vez de evitarlo claramente, como si no considerara que caer es 
igualmente bueno y no bueno? Es evidente, entonces, que cree que una cosa es mejor y la otra no es mejor." (1008 b 14-19). Mediante estos ejemplos Aristóteles intenta probar que, a partir de la observación de la conducta de los agentes, se puede obtener la conclusión de que las creencias relevantes para la acción que éstos poseen no son contradictorias. Se trata, pues, de una inferencia que va de las acciones a las creencias de los sujetos.

El carácter viable de esta argumentación está supeditado al hecho de que tal inferencia sea realizable. A su vez, la posibilidad de efectuar la inferencia depende de ciertos supuestos teóricos acerca de las relaciones que existen entre las acciones y las creencias humanas. Aristóteles, tal como es su costumbre, se expresa de una manera muy elíptica y no hace explícitas todas las premisas de su prueba. Esta es una dificultad del argumento que, no obstante, no resulta insuperable. Acudiendo a sus obras sobre filosofía práctica es posible reconstruir de una manera fiel al pensamiento aristotélico (aunque sin atenerse literalmente a los textos en algunos casos) las tesis principales que sustentan su razonamiento. Los dos supuestos principales de la prueba se refieren al esquema de explicación de la acción y a la racionalidad de la conducta humana.

El deseo (órexis) y la creencia (dóxa) son, según Aristóteles, dos de los componentes necesarios que intervienen en la acción racional, es decir, en aquella que es objeto de elección luego de un proceso de deliberación. Dicha acción, en tanto racional, es la que puede explicarse por medio de razones de actuar del agente. Los deseos y las creencias del agente, que son componentes necesarios de la acción racional, serán naturalmente parte de la explicación de por qué éste realizó una acción determinada. Consideremos, entonces, que la explicación de una acción por medio de razones debe incluir, al menos, el enunciado de un deseo y el de una creencia del agente. Los deseos, según Aristóteles, se refieren siempre a los fines de la acción, o sea, a los tipos de acciones que el agente quiere ejecutar o a 
los estados de cosas que quiere realizar. Aristóteles no es del todo claro y preciso en la determinación del contenido de las creencias relevantes para la acción, pero, podemos aceptar que éstas se refieren a los medios por los cuales se alcanza el fin, o expresan, en general, una condición para ello. ${ }^{1}$ Con estos dos elementos se tiene ya un esquema básico de explicación de las acciones. El esquema explicativo consta de una premisa que enuncia el fin del agente, es decir, que desea un estado de cosas o que se propone ejecutar una clase de acción. En general, decimos que esta premisa expresa el deseo (querer, etc.) del agente. La segunda premisa enuncia la creencia del agente. Esta creencia es relevante para la explicación de una acción determinada cuando afirma la existencia de una conexión entre esa acción y el deseo del agente. El modo típico de esa conexión es el que dice que ejecutar cierta clase de acción es una condicion (necesaria y/o suficiente) para lograr el fin que el agente se propone. ${ }^{2}$

1 Los textos aristotélicos más importantes para la explicación de la acción son: Eth. Nicom. III, 1-5; Eth. Eud. II, 7-11; De an. III, 9-11; De motu an. 6-11; y, con ciertas reservas, Mag. mor. I, 10-18. Para el esquema de explicación me he basado fundamentalmente en Eth. Nicom. III, 3 ; y Eth. Eud. II, 10. Aristóteles afirma que tanto el deseo como la creencia se refieren al fin de la acción ( $c f$. Eth. Eud. II, 10, 1226a 13 y ss.). El fin es aquello que se quiere, es decir, el objeto del deseo. La creencia parece ser, sobre todo, acerca de los medios para alcanzar el fin, puesto que la elección proviene de una creencia deliberativa (doxa bouletiké, 1226 b 9), y, como es sabido, la deliberación y la elección tratan acerca de los medios (cf. Eth. Nicom. III, 2, 1111 b 26-30; 3, 1112 b 11-20). El concepto de deseo (órexis) tiene en Aristóteles un sentido muy amplio que incluye cualquier pro-actitud (cf. Eth. Eud. II, 71223 a 25-29). La teoría aristotélica de la acción ha sido objeto de estudios detallados, como los de A. Kenny, Aristotle's Theory of the Will, y D. Charles, Aristotle's Theory of Action, que son indispensables para un análisis más minucioso del tema.

2 Los esquemas de explicación de la acción actualmente más difundidos son los de G. H. Von Wright y D. Davidson, que también apelan a deseos, en el sentido general de pro-actitudes, y creencias ( $c f . \mathrm{G}$. H. Von Wright, Explanation and Understanding, 96 y ss.; D. Davidson, Essays on Actions and Events, 3 y ss.; 83 y ss.). El esquema aristotélico aquí presen- 
En el texto antes citado, Aristóteles no expone los dos elementos que serían necesarios para explicar las acciones con las que ejemplifica su argumento. No obstante, con base en los supuestos anteriores, ambos ejemplos pueden ser dispuestos bajo la forma de esquemas explicativos, en los cuales cada acción resulta explicada mediante un deseo y una creencia. De este modo, el primero de ellos es así:

Deseo llegar a Megara

Creo que caminar hacia Megara es un medio adecuado para llegar allí

Camino hacia Megara

mientras que el segundo tiene esta forma:

Me propongo hacer lo que es bueno para mi salud

Creo que evitar este precipicio es bueno para mi salud

\section{Evito este precipicio}

En ambos casos es necesario reponer alguna premisa que no se halla explícita en el texto, para completar la explicación, pero se lo hace utilizando como guía un esquema explicativo que es de origen evidentemente aristotélico.

Si los ejemplos se interpretan de acuerdo con este modelo de explicación de la acción basado en deseos y creencias, el argumento aristotélico resulta el siguiente: cualquier agente que tenga un fin determinado y ejecute una acción, no tiene creencias contradictorias relevantes para explicar esa acción. Así, según Aristóteles, no ocurre que alguien se encuentre simultáneamente en estas tres condiciones: 1) desee llegar a Megara; 2) camine hacia Megara; y 3) crea que caminar hacia

tado es, sin embargo, más general que éstos, puesto que los esquemas de Von Wright y Davidson resultan especies de éste. 
Megara es un medio adecuado para llegar allí y que caminar hacia Megara no es un medio adecuado para llegar allí. Si se dan las condiciones 1) y 2), no se da 3). Lo mismo vale para las creencias relevantes de cualquier otro ejemplo del mismo tipo, como la que afirma que evitar un precipicio es bueno y no es bueno.

Aristóteles no dice de qué modo se puede inferir que un agente no tiene creencias contradictorias cuando se conocen sus deseos y sus acciones. La interpretación más adecuada de su argumento parece ser la que lo considera como un argumento empírico, basado en la experiencia de múltiples casos del mismo tipo que los presentados en los ejemplos. ${ }^{3}$ La observación repetida de la conducta de diferentes hombres en circunstancias similares muestra que, en todos los casos registrados, cada vez que alguien se propone un fin $F$ ejecuta la acción $A$, pero nunca omite hacer $A$, es decir, no ocurre que no hace $A$ (consideraremos como sinónimos "no hacer $A$ " y "omitir $A$ "). Conociendo el fin $F$ y habiendo observado que los agentes siempre hacen $A$, se concluye que poseen la creencia $C$ (relevante para explicar la acción) y que, a la vez, no poseen la creencia contradictoria de ésta $-C$. Esta conclusión no dice únicamente que los agentes no poseen creencias contradictorias, sino, además, que poseen una determinada creencia relevante para esa acción. Consideraremos estos dos aspectos separadamente.

Aunque un agente cualquiera ejecute siempre la acción $A$ cuando se propone el fin $F$ ino sería posible que creyera tanto $C$ como $-C$ ? La razón para inferir que no tiene creencias contradictorias consiste en que si las tuviera, debería esperarse con igual grado de probabilidad, que en algunas ocasiones haga $A$ y en otras omita hacer $A$. En efecto, la creencia $C$ le indica

3 En la interpretación del argumento y en otras diversas cuestiones sobre teoría de la acción estoy en deuda con las observaciones y comentarios del profesor Carlos Oller. 
que hacer $A$ es una condición (necesaria o suficiente) para obtener el fin $F$; entonces, si el agente realmente quiere $F$, se esperaría que haga $A$. Por otra parte, $-C$ le dice que hacer $A$ no es una condición (ni necesaria, ni suficiente) para lograr $F$; en consecuencia, se esperaría que se abstenga de hacer $A$, es decir, que no haga $A$ (siempre que no intervengan otros fines del agente para los cuales hacer $A$ sea una condición). Si los hombres tuvieran creencias contradictorias, su conducta debería oscilar de tal modo que, ante el mismo tipo de situación, a veces hicieran una acción determinada y otras veces omitieran hacerla. Con base en creencias contradictorias relevantes están igualmente justificadas tanto una acción determinada como su correspondiente omisión. En tal caso, si no intervienen otras circunstancias, sería esperable que el agente ejecutara esa acción con la misma frecuencia con que la omitiera. La experiencia muestra, sin embargo, que siempre que un agente se propone el fin $F$, ejecuta la acción $A$ y nunca omite haccrla. De allí se concluye que no posee a la vez las creencias $C$ y $-C$. Esta inferencia es, obviamente, de tipo inductivo, está basada en un número limitado de experiencias y su conclusión sólo tiene el valor de lo probable.

Podría objetarse, además, que, aunque se acepte la conclusión anterior, no se sigue de ello que el agente tenga la creencia $C$. Sólo se infiere, con cierta probabilidad, que no cree a la vez $C$ y $-C$, pero no se puede afirmar nada acerca de $C$ aisladamente. Un agente podría hacer siempre $A$ cuando se propone el fin $F$, y, no obstante, creer $-C$ en todos los casos, o bien no poseer ninguna creencia relevante para la acción $A$. Ante tales circunstancias, desde el punto de vista adoptado para la explicación de la acción, diríamos que su acción es irracional, porque el agente no tiene una razón que la explique o justifique. En el último caso, porque carece efectivamente de una creencia relevante que es uno de los requisitos necesarios de la explicación por razones. Un sujeto que ejecutara sus acciones sin poseer alguna creencia relevante para éstas sería un agente 
irracional y no podría ejecutar en vista a los fines que se propone porque no establece ninguna conexión entre sus acciones y sus deseos. En el primer caso, el agente posee una creencia relevante, $-C$, pero no actúa de acuerdo con esta creencia. Ello es así porque $-C$ le indica que hacer $A$ no es un medio adecuado para lograr el fin $F$; en consecuencia, el agente tiene una razón para omitir $A$, pero no tiene una razón para hacer $A$. La acción $A$, no resulta, entonces, justificada por la creencia $-C$ y es, en tal sentido, irracional (siempre que no intervengan otras creencias que la racionalicen). Con todo, aún no se sigue ninguna conclusión acerca de la creencia $C$, pues, podría ocurrir que algunos hombres, o todos ellos, actuaran siempre irracionalmente.

Para superar esta objeción es necesario establecer, entonces, que los agentes, cuya conducta se ha observado, actúan racionalmente. Aristóteles afirma reiteradamente que la racionalidad es una condición necesaria de la acción humana, puesto que no considera que actúan aquellos seres que no son racionales, como los seres inanimados, los animales e incluso los niños. ${ }^{4}$ Esta declaración presenta, sin embargo, una ambigüedad. El dominio de la acción queda restringido al de los seres racionales, pero isignifica ello que todos los hombres actúan siempre racionalmente y que sólo ejecutan acciones racionales?

La afirmación de Aristóteles de que sólo actúan los seres que obran racionalmente puede ser interpretada de dos maneras diferentes. La interpretación más fuerte es la que afirma que toda acción humana es ipso facto racional y que, por tanto, no hay

4 “... no decimos que el niño actúa, ni tampoco el animal, sino sólo el que actúa racionalmente (diá logismón práttonta)" (Eth. Eud. II, 8, 1224 a 28-31). “[...] no se dice que actúan ni los (seres) inanimados ni 'os otros (seres) animados fuera del hombre; es claro, entonces, que el hombre es generador de acciones" (Mag. Mor. I, 11, 1187b 8-10). "[...] el hombre es el único de los animales que es principio de ciertas acciones, pues, de ninguno de los otros diríamos que actúa" (Eıh. Eud. II, 6, 1222 b 20-21; cf. también Eıh. Nicom. VI, 2, 1139 a 19-20). 
acciones irracionales. La más débil, en cambio, sostiene que las acciones humanas son racionales, pero no necesariamente, y que, en consecuencia, existen actos irracionales. Ambas interpretaciones son compatibles con lo que Aristóteles sostiene. $\mathrm{Ni}$ los seres inanimados ni los animales son susceptibles de actuar racionalmente (dejaremos a los niños de lado) y por ello no participan de la acción. Los seres humanos son los únicos que pueden hacerlo, pero ello no implica por sí mismo que todos sus actos sean racionales. También podrían cometer actos irracionales, aunque, obviamente, sólo si lo hacen en algunas ocasiones, pues, de otro modo, perderían su condición de seres racionales. Un ser racional no puede, evidentemente, actuar siempre irracionalmente.

La akrasía, incontinencia o debilidad de la voluntad, tiene la apariencia de ser un caso paradigmático en el que la racionalidad práctica resulta violada. La incontinencia consiste en el hecho de que un agente: a) realiza un razonamiento práctico; b) alcanza la conclusión correcta, i. e. "La acción $A$ es buena (o mejor que otras)"; y c) no ejecuta dicha acción, sino otra, obrando intencionalmente en contra de su propia conclusión. La acción incontinente parece ser intencionalmente ejecutada en contra de las propias creencias del agente, pues, éste cree que hacer $A$ es mejor que hacer $B$ y, sin embargo, hace $B$. La conclusión de un razonamiento práctico representa una creencia racional acerca de un curso de acción a seguir; ${ }^{5}$ es la elec-

5 Aristóteles es sumamente ambiguo acerca de la conclusión de un razonamiento práctico. En ocasiones afirma que la conclusión es lisa y llanamente una acción (claramente en De motu an. 7, 701 a 21-22). En otros lugares, la conclusión es una proposición, aunque no queda cliaro si es normativa ("debo hacer $A$ "), valorativa (" $A$ es bueno" o " $A$ es mejor que $B$ "), o resolutiva ("haré A") (cf. De motu an. 7, 701 a 13-16; 701 a 19-21; Eth. Nicom. VII, 3, 1147 a 24-30). Estas ambigüedades de Aristóteles también pueden hallarse en las discusiones contemporáneas acerca del razonamiento práctico (cf. J. Raz, (ed.), Practical Reasoning, 33 y ss.; 46 y ss.; G. W. Barnes, "The Conclusion of Practical Reasoning", en Analysis, 43, 4, (1983), 193-199). 
ción racional del agente. $\mathrm{Al}$ actuar en contra de su conclusión un agente obra irracionalmente, puesto que su acción no es racionalmente elegida. Se puede, entonces, pensar fundadamente, como lo hace D. Davidson, ${ }^{6}$ que toda acción incontinente es en el fondo irracional, pues el agente no tiene una justificación para ejecutarla. Al no tener una razón, su elección no resulta racionalmente explicable.

En su análisis de la akrasía, en Eth. Nicom. VII, 3, Aristóteles no admite de una manera clara y directa la existencia de tales acciones irracionales. Se resiste, en principio, a aceptar que alguien actúe voluntariamente contra su propio conocimiento ( $c f .1147$ a 9-18), que, para el caso, equivale a una simple creencia verdadera ( $c f .1146$ b 23-26). Esta actitud ha permitido sustentar la interpretación tradicional, según la cual Aristóteles se adhiere a una posición de tipo socrático: la akrasía no es posible y el que obre mal lo hace por falta de conocimiento adecuado. Se sigue de ello que no se puede actuar en contra de la propia conclusión, si ésta es el producto de un razonamiento práctico correcto. El incontinente, entonces, nunca alcanza la conclusión correcta, o bien, si lo hace, la olvida a causa de su ignorancia de alguna premisa de la inferencia práctica. En cualquier caso, no se producen acciones que sean a la vez intencionales e irracionales, como lo exige el concepto de incontinencia.

El análisis más minucioso del concepto aristotélico de akrasía ha sido realizado por D. Charles. ${ }^{7}$ Éste se opone a la interpretación socrática de Aristóteles y sostiene que la acción acrática intencional no sólo es posible, sino también explicable dentro de la teoría aristotélica de la acción. El akratés actúa intencionalmente contra el juicio valorativo que representa su elección racional. La acción acrática es, así, una acción autén-

6 "How is the Weakness of the Will Possible", en: Essays on Actions and Events, 42.

7 Aristotle's Theory of Action, 109-196. 
ticamente irracional, aunque sea susceptible de ser racionalmente explicada. Esto es así porque no es una acción racionalmente elegida. ${ }^{8}$ Según Charles, existen dos tipos de akrasía. El primero es aquél en que el akratés no alcanza la conclusión correcta, pese a conocer las premisas adecuadas. La razón de ello es que no "activa" la premisa menor y falla, entonces, en utilizar las premisas que posee para extraer la conclusión correcta. El segundo tipo es aquel en el cual el akratés alcanza la conclusión correcta, pero la conoce de un modo imperfecto. En este caso, obra contra su propia conclusión porque un deseo sensual le impide ponerla en práctica. Su razonamiento práctico concluye correctamente, mas no resulta productivo de acción. El akratés actúa intencionalmente contra su mejor juicio bajo la influencia de la pasión. ${ }^{9} \mathrm{Al}$ hacerlo parece ejecutar una acción irracional. Sin embargo, todo el análisis de Charles, pese a su intención antisocrática, atribuye la incontinencia a cierta carencia de conocimiento. El akratés que alcanza la conclusión correcta no tiene un conocimiento adecuado de ésta y, por tanto, sólo la posee de una manera imperfecta. ${ }^{10} \mathrm{El}$ error bien puede ser una cuestión de conocimiento práctico y no teórico, pero es, como en el socratismo, una falla de cono-. cimiento. Si esto es así, ya no es evidente que el akratés obre contra su propia conclusión. Puesto que no conoce adecuadamente la conclusión, puede decirse que en sentido estricto no la posee. Además, puede replicarse que si poseyera un conocimiento perfecto de tal conclusión, no podría actuar en contra de ella. La interpretación de Charles, en suma, no ha conseguido probar que Aristóteles admita la existencia de acciones irracionales como las que implica la akrasía. Queda, pues, en pie la

${ }^{8}$ Cf. ibid., 115 y 150.

9 Cf. ibid., 126-127.

10 Según las expresiones de Charles, el akratés conoce la conclusión correcta "in an 'off-colour' fashion" (121) o "in a 'deviant way" (127), y es, entonces, "quasi-ignorant of it" (127). 
posibilidad de sostener que Aristóteles adhiere a un postulado fuerte de racionalidad, según el cual toda acción es racional. Probar que lo sostiene efectivamente requiere, por supuesto, un detallado estudio adicional.

$\mathrm{El}$ presupuesto de la racionalidad de los agentes debe ser contado como una de las premisas de la argumentación aristotélica. Si a ésta se le añade la aceptación del esquema que contiene un deseo y una creencia como un modelo general de explicación de la acción, entonces, se obtiene un argumento completo. No obstante, las conclusiones resultantes acerca de la creencia $C$ son muy diferentes según la interpretación de la racionalidad de las acciones que se adopte. Si se admite que toda acción es racional, se sigue que si un agente cualquiera ejecuta la acción $A$ y tiene el fin $F$, también debe poseer la creencia $C$ que es relevante para su acción. Esto se puede determinar $a$ priori por medio del esquema de racionalización de la acción basado en deseos y creencias, siempre y cuando se lo considere como el único esquema necesario y suficiente para racionalizar una acción. Si, en cambio, sólo se adopta el postulado débil de racionalidad y se admiten actos irracionales, nada puede concluirse con necesidad acerca de las creencias de un agente particular. En efecto, los actos observados podrían haber sido, precisamente, los casos en los cuales ese agente obró irracionalmente, sin una creencia relevante para su acción. Cuanto mayor sea el número de observaciones efectuadas, tanto menor será la probabilidad de encontrar en ellas una serie de actos irracionales, pero nunca habrá certeza de que no ha ocurrido alguno. Bajo esta interpretación, entonces, no se puede inferir que el agente posee la creencia $C$.

Consideremos ahora el caso de las creencias contradictorias. La argumentación de Aristóteles no es una prueba que trate de mostrar que es imposible que un agente posea creencias contradictorias. Tampoco intenta demostrar a priori que una acción realizada con base en tales creencias sea irracional. El modelo aristotélico de explicación de la acción exige únicamente que 
el agente tenga como razón de su acto un deseo y una creencia relevante para éste. Si un sujeto tiene el fin $F$, hace $A$ y cree $C \mathrm{y}-C$, ambas condiciones se cumplen, y, desde tal punto de vista, debe decirse que su acción es racional. En efecto, la acción $A$ está justificada por el fin $F$ y la creencia $C$. Lo paradojico de este caso es que la omisión de $A$ también está justificada, esta vez por la creencia $-C$. Bajo creencias contradictorias (y a falta de otras condiciones) está igualmente justificado tanto hacer $A$ como no hacer $A$. Una acción y su correspondiente omisión resultan ambas racionales. No es improbable que esta situación viole algún requisito de racionalidad, por ejemplo, que la eleccion del agente sea irracional, ya que no tiene una razón para decidir ejecutar $A$ en vez de omitirla. Esta cuestión, sin embargo, está más allá del alcance del argumento aristotélico.

La única base para sostener que un agente no tiene creencias contradictorias es la observación repetida de que, en circunstancias similares, siempre que desea el fin $F$ realiza la acción $A$. Puesto que se admite que el agente actúa racionalmente, en el sentido ya señalado, se sabe que debe actuar de acuerdo con sus creencias. Ahora bien, esto se cumple tanto si el agente hace $A$ como si omite ejecutar esta acción. Cuando se ha observado que siempre hace $A$, cabe preguntar porqué, si tiene creencias contradictorias, no omite a veces hacer $A$. Si el agente actúa de acuerdo con sus creencias, debería a veces hacer $A$ y otras veces debería omitirla. El hecho de que en todos los casos observados haya hecho $A$ hace inductivamente probable que ese agente no crea simultáneamente $C$ y $-C$, como ocurre en los ejemplos presentados. Si tuviera creencias contradictorias su comportamiento, según es esperable, sería diferente.

Hay otro sentido en el cual podría decirse que un agente que tiene creencias contradictorias obra de acuerdo con sus creencias. Este sería el caso en el que a la vez y bajo el mismo respecto ejecutara y no ejecutara una misma acción como $A$. Aristóteles no tiene en cuenta esta posibilidad, más aún, en 
la misma descripción de una acción como una instancia del tipo $A$ se halla supuesta la idea de que esa acción no es simultáneamente la omisión de $A$. Al decir que alguien hace $A$ presuponemos que no se abstiene también de hacer $A$. Esto implica que el PNC ya está presupuesto en la descripción de cualquier acción determinada. En efecto, nunca describiríamos la conducta de un agente diciendo que al mismo tiempo y bajo el mismo respecto hace y no hace $A$. Cuando Aristóteles ofrece sus ejemplos de acciones está admitiendo implícitamente que nadie puede caminar hacia Megara y abstenerse a la vez de caminar hacia Megara, o arrojarse en un precipicio y omitir arrojarse en un precipicio. De otro modo, el argumento práctico no podría dar ningún tipo de apoyo al PNC. Esto, sin embargo, no es un defecto de la argumentación en favor del PNC. El que defiende este principio no puede dejar de aplicarlo en algún caso; debe respetarlo siempre, incluso en sus descripciones de las acciones que emplee en sus argumentos. Si el negador del PNC cree que puede refutarlo mostrando que es posible hacer y no hacer $A$, la carga de la prueba corre por su cuenta. El defensor del principio se limitará a señalar la necesidad de que toda descripción de una acción, y de cualquier evento en general, sea consistente.

En conclusión, el argumento práctico que Aristóteles brinda en favor del PNC tiene un carácter diferente del de las otras pruebas presentadas en Metafísica IV, $4 .{ }^{11}$ No intenta, como las demás, mostrar las consecuencias insostenibles que implica la negación de este principio. No es, entonces, una prueba refutativa, indirecta o negativa. Por el contrario, trata de aportar una evidencia positiva en favor del PNC. Su objetivo es mostrar que ningún hombre posee creencias contradictorias; incluso aquellos que rechazan el PNC no actúan consecuentemente con su

11 Me ocupo de este otro tipo de pruebas en "La justificación aristolélica del principio de no contradicción frente al escepticismo radical”, en Revista Latinoamericana de Filosofia, XIV, 3, (1988), 339-345. 
rechazo. La prueba es de carácter práctico, pues apela a la acción humana para extraer de ella un argumento que apoye al PNC. Tal argumento es de tipo empírico, ya que se halla basado en la observación de la conducta de los agentes. Su conclusión, que ningún hombre actúa con base en creencias contradictorias, es, en consecuencia, meramente probable. La prueba, además, se presenta de una manera incompleta, ya que, para poder inferir que un agente no tiene creencias contradictorias, se requieren los supuestos adicionales acerca de la racionalidad humana y acerca del modo de explicar o racionalizar las acciones. Si se aceptan estos supuestos, de acuerdo con la interpretación antes señalada el argumento se completa y la conclusión se vuelve probable. Sin embargo, ésta no tiene certeza alguna. Es posible, en efecto, que algunos de los agentes observados hayan ejecutado en todos los casos la misma acción, aunque tuvieran creencias contradictorias acerca de ella. Incluso todos los agentes podrían haber actuado de ese modo en ciertas circunstancias. Tal situación parece improbable, pero no queda excluida por un argumento empírico como el de Aristóteles. Dentro de estos límites, no obstante, dicho argumento, completado con los supuestos adicionales necesarios, aporta plausibilidad a la tesis de que los hombres no actúan con base en creencias contradictorias.

\section{BIBLIOGRAFÍA}

Aristóteles, De anima, A. Janonne y E. Barbotin (eds.), Les Belles Lettres, París, 2a. ed. 1980.

__., De motu animalium, E. S. Forster (eds.), Heinemann, L.C.L., Londres, 6a. ed. 1983.

—, Metaphysica, W. D. Ross (ed.), Clarendon Press, Oxford, 6a. ed. 1970, 2 vols.

—_, Ethica Nicomachea, H. Rackham (ed.), Heinemann, L.C.L., Londres, 10a. ed. 1982. 
—_, Magna Moralia, G. Cyril Armstrong (ed.), Heinemann, L.C.L., Londres, 7a. ed. 1977.

,Ethica Eudemia, H. Rackham (ed.), Heinemann, L.C.L., Londres, 1942.

Barnes, G. W., “The Conclusion of Practical Reasoning”, en: Analysis, 43, 4, (1983), pp. 193-199.

Cassini, A., "La justificación aristotélica del principio de no contradicción frente al escepticismo radical", en Revista Latinoamericana de Filosofía, XIV, 3 (1988), pp. 339-345.

Charles, D., Aristotle's Theory of Action, Duckworth, Londres, 1984. Davidson, D., Essays on Actions and Events, Clarendon Press, Oxford, 3a. ed. 1985.

Kenny, A., Aristotle's Theory of the Will, Duckworth, Londres, 1979.

Raz, J., (ed.), Practical Reasoning, Oxford University Press, Oxford, 1978.

Von Wright, G. H., Explanation and Understanding, Routledge and Kegan Paul, Londres, 2a. ed. 1975.

Recibido: 9 enero 1990. 


\section{SUMMARY}

The paper considers an Aristotelian argument to justify the principle of non contradiction given in Metaphysics $1008 \mathrm{~b} 12-31$. This is a practical argument, because it refers to human action and its explanation. First, the article outlines the argument, which is shown to be incomplete. Then, it analyses two fundamental assumptions of the argument: a) the explanation of actions; b) the rationality of actions. The first state that all actions can be explained by means of a desire and a relevant belief; the second, that all actions are rational. It affirms that there are Aristotelians grounds to support both assumptions, despite the existence of akrasía or incontinent action. Finally, it shows that the argument, with these two assumptions as additional premises, is complete and it has inductive plausibility.

[A. C.] 